\title{
Ergodic seminorms for commuting transformations and applications
}

\author{
by \\ Bernard Host (Marne-la-Vallée)
}

\begin{abstract}
Recently, T. Tao gave a finitary proof of a convergence theorem for multiple averages with several commuting transformations, and soon thereafter T. Austin gave an ergodic proof of the same result. Although we give here another proof of the same theorem, this is not the main goal of this paper. Our main concern is to provide tools for the case of several commuting transformations, similar to the tools successfully used in the case of a single transformation, with the idea that they may be used in the solution of other problems.
\end{abstract}

\section{Introduction}

1.1. Motivation and context. Recently, T. Tao $[\mathrm{T}]$ proved a convergence result for several commuting transformations:

Theorem (T. Tao). Let $\left(X, \mu, S_{1}, \ldots, S_{d}\right)$ be a system where $S_{1}, \ldots, S_{d}$ are commuting measure preserving transformations of the probability space $(X, \mu)$. Then for every $f_{1}, \ldots, f_{d} \in L^{\infty}(\mu)$, the averages

$$
\frac{1}{N} \sum_{n=0}^{N-1} S_{1}^{n} f_{1} \cdot \ldots \cdot S_{d}^{n} f_{d}
$$

converge in $L^{2}(\mu)$.

For $d=2$ the result was proved by Conze and Lesigne [CL]. The particular case when the transformations $T_{i}$ are powers of a single transformation, for example $T_{i}=T^{i}$, was solved in [HK1].

Tao's proof does not really belong to ergodic theory: it only uses the pointwise ergodic theorem in order to translate the problem into a finitary question. Soon after, H. Towsner [To] rewrote Tao's proof using nonstandard analysis. More recently, T. Austin [A] gave another proof of the same result by more conventional ergodic methods, and the present work was inspired by reading his paper.

2000 Mathematics Subject Classification: 37A05, 37A30.

Key words and phrases: multiple ergodic averages, commuting transformations. 
Let us say a few words about the methods. All the papers dealing with a single transformation use an idea introduced implicitly by Furstenberg $[\mathrm{F}]$ : the construction of a characteristic factor. It is a factor (i.e. a quotient) of the system controlling the asymptotic behavior of the multiple averages so that one can restrict the study only to functions defined on this factor. The next step is to prove that this factor has some useful structure, and, once this is done, convergence is generally easier to prove. In short, the convergence follows from the existence of some hidden structure in the system. The structure given in [HK1] can be used to study other problems of multiple convergence and of multiple recurrence, like for example in [HK2], [L], [BHK], [FHK], [FK2]. A similar method was used by Conze and Lesigne for two commuting transformations, but all attempts to solve the general case using the machinery of characteristic factors have been unsuccessful $\left(^{1}\right)$.

T. Austin proceeds in the opposite direction, building an extension of the original system with good properties; he calls this extension a pleasant system. This extension is not very explicit (it is defined as an inverse limit) and it gives little information about the original system. Moreover, its construction is directly related to the averages (1) and seems to be difficult to use for related problems.

Although here we give a fourth proof of Tao's result, this is not the main point of this paper. Our main goal is to provide tools for the case of several commuting transformations, similar to the tools successfully used in the case of a single transformation, with the idea that they can be useful in the solution of other problems. For this reason, we conclude this paper by giving some properties that we do not immediately need (see Section 4), but should be useful in other applications.

The price to pay for more generality is that some proofs in this paper are less elementary than those in Austin's.

1.2. Tao's method gives the convergence of the ordinary averages (1) only, while Austin's proof, as well as ours, generalizes to "uniform averages":

Theorem 1 (T. Austin). Let $\left(X, \mu, S_{1}, \ldots, S_{d}\right)$ be a system where $S_{1}, \ldots, S_{d}$ are commuting measure preserving transformations. Then for all $f_{1}, \ldots, f_{d} \in L^{\infty}(\mu)$, the averages

$$
\frac{1}{\left|I_{j}\right|} \sum_{n \in I_{j}} S_{1}^{n} f_{1} \cdot \ldots \cdot S_{d}^{n} f_{d}
$$

converge in $L^{2}(\mu)$ for any sequence $\left(I_{j}: j \geq 1\right)$ of intervals in $\mathbb{Z}$ whose lengths $\left|I_{j}\right|$ tend to infinity.

$\left({ }^{1}\right)$ The case considered in [FK1] is very particular. 
In fact, Austin's result is slightly more general: instead of commuting transformations he considers commuting measure preserving $\mathbb{Z}^{r}$-actions on $X$; the averages on intervals are replaced by averages on a Følner sequence in $\mathbb{Z}^{r}$. Up to minor changes (almost only in notation), the method presented here can be used in this more general situation. For simplicity, we restrict ourselves to the case stated in Theorem 1.

1.3. Contents. We first follow the same strategy as in the first sections of [HK1]: given a system $\left(X, \mu, T_{1}, \ldots, T_{d}\right)$ where the transformations commute, in Section 2 we build a measure $\mu^{*}$ on some Cartesian (finite) power $X^{*}$ of $X$ and use it to define a seminorm $\|\cdot\|$ on $L^{\infty}(\mu)$. Then we establish the properties used in the proof of Tao's theorem. We show:

Proposition 1. Let $\left(X, \mu, S_{1}, \ldots, S_{d}\right)$ be a system where $S_{1}, \ldots, S_{d}$ are commuting measure preserving transformations. Define $T_{1}=S_{1}$ and $T_{i}=$ $S_{i} S_{1}^{-1}$ for $2 \leq i \leq d$ and let $\|\cdot\|$ denote the seminorm on $L^{\infty}(\mu)$ associated to the system $\left(X, \mu, T_{d}, \ldots, T_{2}, T_{1}\right)$. Then for all $f_{1}, \ldots, f_{d} \in L^{\infty}(\mu)$ with $\left\|f_{i}\right\|_{L^{\infty}(\mu)} \leq 1$ for $2 \leq i \leq d$, we have

$$
\limsup _{j \rightarrow \infty}\left\|\frac{1}{\left|I_{j}\right|} \sum_{n \in I_{j}} S_{1}^{n} f_{1} \cdot \ldots \cdot S_{d}^{n} f_{d}\right\|_{L^{2}(\mu)} \leq\left\|f_{1}\right\|
$$

for every sequence $\left(I_{j}: j \geq 1\right)$ of intervals in $\mathbb{Z}$ whose lengths tend to infinity.

Next, we remark that $X^{*}$ is naturally endowed with some commuting transformations $T_{1}^{*}, \ldots, T_{d}^{*}$ and that $X^{*}$, endowed with $\mu^{*}$ and with these transformations, admits $X$ as a factor. Therefore, in order to prove the convergence of the averages (1), we can substitute $X^{*}$ for $X$.

Properties of this system are established in Section 3. Substituting $\left(X^{*}, \mu^{*}, T_{1}^{*}, \ldots, T_{d}^{*}\right)$ for $\left(X, \mu, T_{1}, \ldots, T_{d}\right)$, we define a seminorm $\|\cdot\|^{*}$ on $L^{\infty}\left(\mu^{*}\right)$. Before stating our main result we introduce notation used throughout this paper.

Notation. If $S$ is a measure preserving transformation of a probability space $(Y, \nu)$, we write $\mathcal{I}(S)$ for the $\sigma$-algebra of $S$-invariant sets.

Theorem 2. Let $\mathcal{W}^{*}$ be the $\sigma$-algebra

$$
\mathcal{W}^{*}:=\bigvee_{i=1}^{d} \mathcal{I}\left(T_{i}^{*}\right)
$$

of $\left(X^{*}, \mu^{*}\right)$. If $F \in L^{\infty}\left(\mu^{*}\right)$ is such that $\mathbb{E}_{\mu^{*}}\left(F \mid \mathcal{W}^{*}\right)=0$, then $\|F\|^{*}=0$.

We call a system with this property a magic system.

Theorem 2 implies in particular that every system has a magic extension. This notion is similar to that of a pleasant system in $[\mathrm{A}]$ and is used in the 
same way. The differences are that $X^{*}$ is a relatively explicit system $\left({ }^{2}\right)$ ( $X^{*}$ is a finite Cartesian power of $X$ ) and that its construction is related to the seminorm associated to the transformations, and not only to the averages (1). Therefore it can be used to study any other question involving this seminorm.

Tao's ergodic theorem follows easily from the preceding two results:

Proof of Theorem 1, assuming everything above. We proceed by induction on $d$. For $d=1$, the statement is the mean ergodic theorem. We take $d>1$ and assume that the result holds for $d-1$ transformations.

Let $T_{1}, \ldots, T_{d}$ and $\|\cdot\|$ be as in Proposition $1,\left(X^{*}, \mu^{*}, T_{1}^{*}, \ldots, T_{d}^{*}\right)$ as above, and $\mathcal{W}^{*}$ as in Theorem 2 . We define transformations $S_{1}^{*}, \ldots, S_{d}^{*}$ of $X^{*}$ by $S_{1}^{*}=T_{1}^{*}$ and $S_{i}^{*}=T_{i}^{*} T_{1}^{*-1}$ for $2 \leq i \leq d$.

We see that $\left(X, \mu, S_{1}, \ldots, S_{d}\right)$ is a factor of $\left(X^{*}, \mu^{*}, S_{1}^{*}, \ldots, S_{d}^{*}\right)$. Therefore, in order to prove the convergence of the averages $(2)$ in $L^{2}(\mu)$ for functions $f_{1}, \ldots, f_{d}$ in $L^{\infty}(\mu)$, it suffices to show the convergence in $L^{2}\left(\mu^{*}\right)$ of the averages

$$
\frac{1}{\left|I_{j}\right|} \sum_{n \in I_{j}} S_{1}^{* n} f_{1}^{*} \cdot \ldots \cdot S_{d}^{* n} f_{d}^{*}
$$

for functions $f_{1}^{*}, \ldots, f_{d}^{*}$ in $L^{\infty}\left(\mu^{*}\right)$.

Consider first the case that

$$
f_{1}^{*}=g_{2} \cdots g_{d} \quad \text { where } g_{i} \text { is invariant under } T_{i}^{*} \text { for } 2 \leq i \leq d .
$$

Since $T_{i}^{*}=S_{i}^{*} S_{1}^{*-1}$ for $2 \leq i \leq d$, the averages (3) can be rewritten as

$$
\frac{1}{\left|I_{j}\right|} \sum_{n \in I_{j}} S_{2}^{* n}\left(g_{2} f_{2}^{*}\right) \cdot \ldots \cdot S_{d}^{* n}\left(g_{d} f_{d}^{*}\right)
$$

and the convergence in $L^{2}\left(\mu^{*}\right)$ follows from the induction hypothesis.

Since the linear span of the functions of the form (4) is dense in $L^{\infty}\left(\mu^{*}, \mathcal{W}^{*}\right)$ under the norm of $L^{1}\left(\mu^{*}\right)$, by density the averages (3) converge whenever the function $f_{1}^{*}$ is measurable with respect to $\mathcal{W}^{*}$.

We are left with checking the case that $\mathbb{E}_{\mu^{*}}\left(f_{1}^{*} \mid \mathcal{W}^{*}\right)=0$. We have $\left\|f_{1}^{*}\right\|^{*}=0$ by Theorem 2 and the averages (3) converge to 0 in $L^{2}\left(\mu^{*}\right)$ by Proposition 1 applied to the system $\left(X^{*}, \mu^{*}, S_{1}^{*}, \ldots, S_{d}^{*}\right)$.

2. The box measure and the box seminorm. The objects defined in this section, as well as their properties, are completely similar to those of Section 3 of [HK1]. Most of the proofs are exactly the same and we only sketch them.

$\left({ }^{2}\right)$ It seems plausible that the methods used here in the proof of Theorem 2 can be combined with the constructions of $[\mathrm{A}]$, removing the need for the inverse limit. 
2.1. Notation. All functions are implicitly assumed to be measurable and real-valued.

If $S$ is a measure preserving transformation of a probability space $(Y, \nu)$, the conditionally independent square of $\nu$ over the $\sigma$-algebra $\mathcal{I}(S)$ is the measure $\nu \times_{\mathcal{I}(S)} \nu$ on $Y \times Y$ characterized by: for all bounded measurable functions $f, f^{\prime}$ on $X$,

$$
\int f(y) f^{\prime}\left(y^{\prime}\right) d\left(\nu \times_{\mathcal{I}(S)} \nu\right)\left(y, y^{\prime}\right)=\int \mathbb{E}_{\nu}(f \mid \mathcal{I}(S)) \mathbb{E}_{\nu}\left(f^{\prime} \mid \mathcal{I}(S)\right) d \nu .
$$

We write $X^{*}=X^{2^{d}}$. We introduce some conventions for notation of points in this space and more generally in $X^{2^{k}}$ where $k \geq 1$ is an integer.

The points of $X^{2^{k}}$ are written as

$$
x=\left(x_{\epsilon}: \epsilon \in\{0,1\}^{k}\right) .
$$

Each $\epsilon \in\{0,1\}^{k}$ is written without commas or parentheses. If $k \geq 2$ and $\eta \in\{0,1\}^{k-1}$, we write $\eta 0=\eta_{1} \ldots \eta_{k-1} 0$ and $\eta 1=\eta_{1} \ldots \eta_{k-1} 1$.

Occasionally, it is also convenient to use another notation. We write $[k]=\{1, \ldots, k\}$ and make the natural identification between $\{0,1\}^{k}$ and the family of subsets of $[k]$. Therefore, for $\epsilon \in\{0,1\}^{k}$ and $1 \leq i \leq k$, the assertion " $\epsilon_{i}=1$ " is equivalent to " $i \in \epsilon$ ". Therefore we write $\emptyset=0 \ldots 0 \in\{0,1\}^{k}$.

If $f_{\epsilon}, \epsilon \in\{0,1\}^{k}$, are functions on $X$, we define a function on $X^{2^{k}}$ by

$$
\left(\bigotimes_{\epsilon \in\{0,1\}^{k}} f_{\epsilon}\right)(x):=\prod_{\epsilon \in\{0,1\}^{k}} f_{\epsilon}\left(x_{\epsilon}\right) .
$$

For $1 \leq i \leq d, T_{i}^{\Delta}$ denotes the diagonal transformation $T_{i} \times \cdots \times T_{i}$ of $X^{2^{k}}$ :

$$
\left(T_{i}^{\Delta} x\right)_{\epsilon}=T_{i} x_{\epsilon} \quad \text { for every } \epsilon \in\{0,1\}^{d},
$$

and the side transformations $T_{i}^{*}$ of $X^{*}$ are defined by

$$
\left(T_{i}^{*} x\right)_{\epsilon}=\left\{\begin{array}{ll}
T_{i} x_{\epsilon} & \text { if } \epsilon_{i}=0, \\
x_{\epsilon} & \text { if } \epsilon_{i}=1,
\end{array} \quad \text { for every } \epsilon \in\{0,1\}^{d} .\right.
$$

2.2. The box measure. We build a measure $\mu^{*}$ on $X^{*}$. First we define a measure $\mu_{T_{1}}$ on $X^{2}$ by

$$
\mu_{T_{1}}=\mu \times_{\mathcal{I}\left(T_{1}\right)} \mu .
$$

This means that for $f_{0}, f_{1} \in L^{\infty}(\mu)$, we have

$$
\int f_{0}\left(x_{0}\right) f_{1}\left(x_{1}\right) d \mu_{T_{1}}(x)=\int \mathbb{E}\left(f_{0} \mid \mathcal{I}\left(T_{1}\right)\right) \mathbb{E}\left(f_{1} \mid \mathcal{I}\left(T_{1}\right)\right) d \mu .
$$

This measure is invariant under the transformations

$$
T_{i} \times T_{i} \quad(1 \leq i \leq d) \quad \text { and } \quad T_{1} \times \mathrm{Id} .
$$

Next we define the measure $\mu_{T_{1}, T_{2}}$ on $X^{4}=X^{2} \times X^{2}$ by

$$
\mu_{T_{1}, T_{2}}=\mu_{T_{1}} \times_{\mathcal{I}\left(T_{2} \times T_{2}\right)} \mu_{T_{1}} .
$$


This means that for $f_{00}, \ldots, f_{11} \in L^{\infty}(\mu)$ we have

$$
\begin{aligned}
& \int \prod_{\epsilon \in\{0,1\}^{2}} f_{\epsilon}\left(x_{\epsilon}\right) d \mu_{T_{1}, T_{2}}(x) \\
& \quad=\int \mathbb{E}_{\mu_{T_{1}}}\left(f_{00} \otimes f_{10} \mid \mathcal{I}\left(T_{2} \times T_{2}\right)\right) \mathbb{E}_{\mu_{T_{1}}}\left(f_{01} \otimes f_{11} \mid \mathcal{I}\left(T_{2} \times T_{2}\right)\right) d \mu_{T_{1}} .
\end{aligned}
$$

For $1 \leq i \leq d$, this measure is invariant under the "diagonal transformations" $T_{i} \times T_{i} \times T_{i} \times T_{i}$ of $X^{4}$; it is also invariant under the "side transformations" $T_{1} \times \mathrm{Id} \times T_{1} \times \mathrm{Id}$ and $T_{2} \times T_{2} \times \mathrm{Id} \times \mathrm{Id}$.

In the same way, for $k<d$ we obtain a measure $\mu_{T_{1}, \ldots, T_{k}}$ on $X^{2^{k}}$, invariant under all "diagonal transformations" $T_{i} \times \cdots \times T_{i}(1 \leq i \leq d)$ and under the "side transformations" associated to $T_{1}, \ldots, T_{k}$ as in (5), but with $k$ substituted for $d$. We define

$$
\mu_{T_{1}, \ldots, T_{k+1}}=\mu_{T_{1}, \ldots, T_{k}} \times_{\mathcal{I}\left(T_{k+1} \times T_{k+1} \times \cdots \times T_{k+1}\right)} \mu_{T_{1}, \ldots, T_{k}} .
$$

After $d$ steps, we obtain a measure $\mu^{*}:=\mu_{T_{1}, \ldots, T_{d}}$ on $X^{*}=X^{2^{d}}$. For $f_{\epsilon} \in L^{\infty}(\mu), \epsilon \in\{0,1\}^{d}$, we have

$$
\begin{aligned}
\int \bigotimes_{\epsilon \in\{0,1\}^{d}} f_{\epsilon} d \mu^{*}(x) \\
=\int \mathbb{E}_{\mu_{T_{1}, \ldots, T_{d-1}}}\left(\bigotimes_{\eta \in\{0,1\}^{d-1}} f_{\eta 0} \mid \mathcal{I}\left(T_{d} \times \cdots \times T_{d}\right)\right) \\
\quad \cdot \mathbb{E}_{\mu_{T_{1}, \ldots, T_{d-1}}}\left(\bigotimes_{\eta \in\{0,1\}^{d-1}} f_{\eta 1} \mid \mathcal{I}\left(T_{d} \times \cdots \times T_{d}\right)\right) d \mu_{T_{1}, \ldots, T_{d-1}}
\end{aligned}
$$

and thus

$$
\int \bigotimes_{\epsilon \in\{0,1\}^{d}} f_{\epsilon} d \mu^{*}(x)=\lim _{N \rightarrow \infty} \frac{1}{N} \sum_{n=0}^{N-1} \int \bigotimes_{\eta \in\{0,1\}^{d-1}}\left(T_{d}^{n} f_{\eta 0} \cdot f_{\eta_{1}}\right) d \mu_{T_{1}, \ldots, T_{d-1}} .
$$

Moreover, the same convergence holds if the intervals $[0, N)$ are replaced by any sequence of intervals whose lengths tend to infinity. Starting from (8) and proceeding by downward induction we get:

Lemma 1. For $f_{\epsilon} \in L^{\infty}(\mu), \epsilon \in\{0,1\}^{d}$, we have

$$
\begin{aligned}
& \int \prod_{\epsilon \in\{0,1\}^{d}} f_{\epsilon}\left(x_{\epsilon}\right) d \mu^{*}(x) \\
& =\lim _{N_{d} \rightarrow \infty} \frac{1}{N_{d}} \sum_{n_{d}=0}^{N_{d}-1} \cdots \lim _{N_{2} \rightarrow \infty} \frac{1}{N_{2}} \sum_{n_{2}=0}^{N_{2}-1} \lim _{N_{1} \rightarrow \infty} \frac{1}{N_{1}} \sum_{n_{1}=0}^{N_{1}-1} \\
& \int \prod_{\epsilon \in\{0,1\}^{d}}^{T_{1}^{\left(1-\epsilon_{1}\right) n_{1}} \ldots T_{d}^{\left(1-\epsilon_{d}\right) n_{d}} f_{\epsilon} d \mu .}
\end{aligned}
$$


Moreover, relation (9) holds for averages on any other sequence of intervals whose lengths tend to infinity, for example for the symmetric averages on $\left[-N_{i}, N_{i}\right]$.

The measure $\mu^{*}$ is invariant under the diagonal transformations $T_{i}^{\Delta}$ and the side transformations $T_{i}^{*}, 1 \leq i \leq d$. This measure is called the box measure associated to the transformations $T_{1}, \ldots, T_{d}$.

In some cases, we write $\mu_{T_{1}, \ldots, T_{d}}$ instead of $\mu^{*}$ to avoid any possible ambiguity.

We notice that all the marginals of $\mu^{*}$ are equal to $\mu$ and that the projection $\pi_{\emptyset}: X^{2^{k}} \rightarrow X$ given by $\pi_{\emptyset}(x)=x_{\emptyset}$ is a factor map from $\left(X^{*}, \mu^{*}, T_{1}^{*}, \ldots, T_{d}^{*}\right)$ to $\left(X, \mu, T_{1}, \ldots, T_{d}\right)$.

For $1 \leq i \leq d$, the coordinate indexed by any $\epsilon \in\{0,1\}^{d}$ plays the same role in the construction of $\mu^{*}$ as the coordinate indexed by $\epsilon^{\prime}$ obtained by substituting $1-\epsilon_{i}$ for $\epsilon_{i}$. This shows that the measure $\mu^{*}$ is invariant under the symmetry of $X^{*}$ associated to this map in the obvious way.

2.3. The box seminorm. By (7), for every $f \in L^{\infty}(\mu)$ we have

$$
\int \prod_{\epsilon \in\{0,1\}^{d}} f\left(x_{\epsilon}\right) d \mu^{*}(x) \geq 0
$$

and we can define:

Definition. For $f \in L^{\infty}(\mu)$,

$$
\|f\|:=\left(\int \prod_{\epsilon \in\{0,1\}^{d}} f\left(x_{\epsilon}\right) d \mu^{*}(x)\right)^{1 / 2^{d}} .
$$

When needed, we write $\|f\|_{T_{1}, \ldots, T_{d}}$ instead of $\|f\|$.

From (8) we get:

For every $f \in L^{\infty}(\mu)$ we have

$$
\|f\|_{T_{1}, \ldots, T_{d}}^{2^{d}}=\lim _{N_{d} \rightarrow \infty} \sum_{n_{d}=0}^{N_{d}-1}\left\|T_{d}^{n_{d}} f \cdot f\right\|_{T_{1}, \ldots, T_{d-1}}^{2^{d-1}} .
$$

Proposition 2 (and definition).

(i) For $f_{\epsilon} \in L^{\infty}(\mu), \epsilon \in\{0,1\}^{d}$, we have

$$
\left|\int \bigotimes_{\epsilon \in\{0,1\}^{d}} f_{\epsilon} d \mu^{*}\right| \leq \prod_{\epsilon \in\{0,1\}^{d}}\left\|f_{\epsilon}\right\|
$$

(ii) \|\|$\cdot \|$ is a seminorm on $L^{\infty}(\mu)$. We call it the box seminorm associated to $T_{1}, \ldots, T_{d}$. 
The bound (12) is similar to the Cauchy-Schwarz-Gowers Inequality.

Proof. The first part of Proposition 2 is proved by induction on $d$. For $d=1$, the result follows immediately from the definition (6) of $\mu_{T_{1}}$ and the Cauchy-Schwarz Inequality. We assume now that $d \geq 2$ and that the result is true for $d-1$ transformations.

For $\epsilon \in\{0,1\}^{d}$ we define two functions $f_{\epsilon}^{\prime}$ and $f_{\epsilon}^{\prime \prime}$ on $X$ by

$$
f_{\eta 0}^{\prime}=f_{\eta 1}^{\prime}=f_{\eta 0} \quad \text { and } \quad f_{\eta 0}^{\prime \prime}=f_{\eta 1}^{\prime \prime}=f_{\eta 1} \quad \text { for all } \eta \in\{0,1\}^{d-1} .
$$

Let $I$ be the left hand side of (12) and let $I^{\prime}$ and $I^{\prime \prime}$ be, respectively, the similar expressions obtained by substituting the functions $f_{\epsilon}^{\prime}$, respectively $f_{\epsilon}^{\prime \prime}$, for the functions $f_{\epsilon}$. By (7) and the Cauchy-Schwarz Inequality, we have $I^{2} \leq I^{\prime} I^{\prime \prime}$.

By (8), the induction hypothesis, the Hölder Inequality, and (11),

$$
\begin{aligned}
I^{\prime} & =\left|\lim _{N_{d} \rightarrow \infty} \frac{1}{N_{d}} \sum_{n_{d}=0}^{N_{d}-1} \int_{\eta \in\{0,1\}^{d-1}}\left(T_{d}^{n} f_{\eta 0} \cdot f_{\eta_{0}}\right) d \mu_{T_{1}, \ldots, T_{d-1}}\right| \\
& \leq \limsup _{N_{d} \rightarrow \infty} \frac{1}{N_{d}} \sum_{n_{d}=0}^{N_{d}-1} \prod_{\eta \in\{0,1\}^{d-1}}\left\|T_{d}^{n_{d}} f_{\eta 0} \cdot f_{\eta 0}\right\|_{T_{1}, \ldots, T_{d-1}} \leq \prod_{\eta \in\{0,1\}^{d-1}}\left\|f_{\eta 0}\right\|_{T_{1}, \ldots, T_{d}}^{2} .
\end{aligned}
$$

A similar bound holds for $I^{\prime \prime}$ and the result follows.

The second part of the proposition is obtained by using the same proof as for Lemma 3.9 in [HK1].

2.4. Proof of Proposition 1. The proof is the same as in the case of a single transformation; see for example Theorem 12.1 in [HK1]. The proof proceeds by induction on $d$. For $d=1$ the seminorm is the absolute value of the integral and there is nothing to prove. Assume $d>1$ and that the result is true for $d-1$ transformations.

Let $f_{1}, \ldots, f_{d}$ and $S_{1}, \ldots, S_{d}$ be as in the statement. We recall that $T_{1}=$ $S_{1}$ and that $T_{i}=S_{i} S_{1}^{-1}$ for $2 \leq i \leq d$. By the van der Corput Lemma and Cauchy-Schwarz Inequality, the limsup in the proposition is bounded by

$$
\limsup _{H \rightarrow \infty} \frac{1}{H} \sum_{h=0}^{H-1} \limsup _{j \rightarrow \infty}\left\|\frac{1}{\left|I_{j}\right|} \sum_{n \in I_{j}} \prod_{\substack{1 \leq i \leq d \\ i \neq 2}}\left(S_{i} S_{2}^{-1}\right)^{n}\left(f_{i} \cdot S_{i}^{h} f_{i}\right)\right\|_{L^{2}(\mu)} .
$$

By the induction hypothesis, this limsup is bounded by

$$
\limsup _{H \rightarrow \infty} \frac{1}{H} \sum_{h=0}^{H-1}\left\|f_{1} \cdot S_{1}^{h} f_{1}\right\|^{\sharp},
$$

where $\|\cdot\| \|^{\sharp}$ is the seminorm associated to the transformations $\left(S_{d} S_{2}^{-1}\right)\left(S_{1} S_{2}^{-1}\right)^{-1}=T_{d}, \ldots,\left(S_{3} S_{2}^{-1}\right)\left(S_{1} S_{2}^{-1}\right)^{-1}=T_{3} \quad$ and $\quad S_{1} S_{2}^{-1}=T_{2}^{-1}$. 
By construction, this seminorm remains unchanged if $T_{2}$ is substituted for $T_{2}^{-1}$ and thus is equal to the seminorm $\|\cdot\|_{T_{d}, \ldots, T_{3}, T_{2}}$.

By Lemma 1 and by Corollary 3 (see Section 4),

$$
\frac{1}{H} \sum_{h=0}^{H-1}\left\|f_{1} \cdot S_{1}^{h} f_{1}\right\|_{T_{d}, \ldots, T_{2}}^{2^{d-1}} \rightarrow\left\|f_{1}\right\|_{T_{d}, \ldots, T_{2}, S_{1}}^{2^{d}} \quad \text { as } H \rightarrow \infty .
$$

Since $S_{1}=T_{1}$, the proof is complete.

2.5. A uniformity result. The next lemma has no analog in [HK1].

Lemma 2. Let $f_{\emptyset} \in L^{\infty}(\mu)$. For every $\delta>0$, there exists $N_{0}=N_{0}(\delta)$ such that for all $f_{\epsilon} \in L^{\infty}(\mu), \emptyset \neq \epsilon \in\{0,1\}^{d}$ with $\left\|f_{\epsilon}\right\|_{L^{\infty}(\mu)} \leq 1$, and all intervals $I_{1}, \ldots, I_{d}$ of $\mathbb{Z}$ of length $\geq N_{0}$, we have

$$
\left|\frac{1}{\left|I_{1}\right| \ldots\left|I_{d}\right|} \sum_{\substack{n_{1} \in I_{1} \\ n_{d} \in I_{d}}} \int \prod_{\epsilon \in\{0,1\}^{d}} T_{1}^{\left(1-\epsilon_{1}\right) n_{1}} \ldots T_{d}^{\left(1-\epsilon_{d}\right) n_{d}} f_{\epsilon} d \mu\right|<\left\|f_{\emptyset}\right\|+\delta .
$$

Proof. We can assume that $\left\|f_{\emptyset}\right\|_{L^{\infty}(\mu)} \leq 1$.

Let $J$ be the average in the statement and let $H_{1}, \ldots, H_{d}$ be integers with $1 \leq H_{i} \leq\left|I_{i}\right|$ for all $i$.

Each $\epsilon \in\{0,1\}^{d}$ is written either as $\epsilon=\eta 0$ with $\eta \in\{0,1\}^{d-1}$ or as $\epsilon=\eta 1$, depending on the value of $\epsilon_{d}$. We split the product in the integral into two parts:

(i) The product of the terms indexed by $\eta 0$ for some $\eta \in\{0,1\}^{d-1}$. This product can be written as $T_{d}^{n_{d}} F_{n_{1}, \ldots, n_{d-1}}$.

(ii) The product $F_{n_{1}, \ldots, n_{d-1}}^{\prime}$ of the terms indexed by $\eta 1$ for some $\eta \in$ $\{0,1\}^{d-1}$.

Thus $J$ is equal to

$$
\frac{1}{\left|I_{1}\right| \ldots\left|I_{d-1}\right|} \sum_{\substack{n_{1} \in I_{1} \\ \cdots \\ n_{d-1} \in I_{d-1}}} \int \frac{1}{\left|I_{d}\right|} \sum_{n_{d} \in I_{d}} T^{n_{d}} F_{n_{1}, \ldots, n_{d-1}} \cdot F_{n_{1}, \ldots, n_{d-1}}^{\prime} d \mu .
$$

Since $\left|F_{n_{1}, \ldots, n_{d-1}}^{\prime}\right| \leq 1$, we have

$$
|J|^{2} \leq \frac{1}{\left|I_{1}\right| \ldots\left|I_{d-1}\right|} \sum_{\substack{n_{1} \in I_{1} \\ \cdots \cdots \\ n_{d-1} \in I_{d-1}}}\left\|\frac{1}{\left|I_{d}\right|} \sum_{n_{d} \in I_{d}} T^{n_{d}} F_{n_{1}, \ldots, n_{d-1}}\right\|_{L^{2}(\mu)}^{2} .
$$

By the finite van der Corput Lemma, the square of the norm in this formula is bounded by the absolute value of

$$
\frac{4 H_{d}}{\left|I_{d}\right|}+\sum_{h_{d}=-H_{d}}^{H_{d}} \frac{H_{d}-\left|h_{d}\right|}{H_{d}^{2}} \int T^{h_{d}} F_{n_{1}, \ldots, n_{d-1}} \cdot F_{n_{1}, \ldots, n_{d-1}} d \mu .
$$


Replacing $F$ by its value shows that $|J|^{2}$ is bounded by the absolute value of

$$
\begin{aligned}
& \frac{4 H_{d}}{\left|I_{d}\right|}+\frac{1}{\left|I_{1}\right| \ldots\left|I_{d-1}\right|} \sum_{\substack{n_{1} \in I_{1} \\
\ldots, \ldots \\
n_{d-1} \in I_{d-1}}} \sum_{h_{d}=-H_{d}}^{H_{d}} \frac{H_{d}-\left|h_{d}\right|}{H_{d}^{2}} \\
& \quad \times \int \prod_{\epsilon \in\{0,1\}^{d}} T_{1}^{\left(1-\epsilon_{1}\right) n_{1}} \ldots T_{d-1}^{\left(1-\epsilon_{d-1}\right) n_{d-1}} T_{d}^{\left(1-\epsilon_{d}\right) h_{d}} g_{\epsilon} d \mu,
\end{aligned}
$$

where the functions $g_{\epsilon}$ are given by $g_{\eta 0}=g_{\eta 1}=f_{\epsilon}$ for $\eta \in\{0,1\}^{d-1}$.

We iterate the same computation, using successively $\epsilon_{d-1}, \ldots, \epsilon_{2}, \epsilon_{1}$ instead of $\epsilon_{d}$. Then

$$
\begin{aligned}
|J|^{2^{d}} \leq & C\left(\frac{H_{1}}{\left|I_{1}\right|}+\cdots+\frac{H_{d}}{\left|I_{d}\right|}\right) \\
+ & \left|\sum_{\substack{-H_{1} \leq h_{1} \leq H_{1} \\
-H_{d} \leq h_{d} \leq H_{d}}} \prod_{i=1}^{d} \frac{H_{i}-\left|h_{i}\right|}{H_{i}^{2}} \int \prod_{\epsilon \in\{0,1\}^{d}} T_{1}^{\left(1-\epsilon_{1}\right) h_{1}} \ldots T_{d}^{\left(1-\epsilon_{d}\right) h_{d}} f_{\emptyset} d \mu\right|
\end{aligned}
$$

for some absolute constant $C$.

By Lemma 1, the iterated limit of the last average when $H_{1} \rightarrow \infty, \ldots$, $H_{d} \rightarrow \infty$ is equal to $\left\|f_{\emptyset}\right\|^{2^{d}}$. Therefore there exist $H_{1}, \ldots, H_{d}$ such that this average has absolute value less than $\left(\left\|f_{\emptyset}\right\|+\delta / 2\right)^{2^{d}}$. The result follows.

REMARK 1. Corollary 3 (Section 4) follows easily from Lemmas 1 and 2 and thus does not depend on Proposition 3. Moreover, it is easy to check that the role played by $f_{\emptyset}$ in Lemma 2 can be played by $f_{\eta}$ for any $\eta \in\{0,1\}^{d}$ and this implies a weak version of the bound (12) in Proposition 2: the integral of the left hand side is equal to zero whenever at least one of the functions $f_{\epsilon}$ has zero seminorm. In fact, this weak version would suffice for our purpose.

2.6. A characteristic $\sigma$-algebra on $X$. The definitions and results of this section are completely similar to those of Section 4.2 of [HK1].

Let us identify $X^{*}=X^{2^{d}}$ with $X^{2^{d-1}} \times X^{2^{d-1}}$; each point $x \in X^{*}$ is written $x=\left(x^{\prime}, x^{\prime \prime}\right)$, where $x^{\prime}, x^{\prime \prime} \in X^{2^{d-1}}$ are given by

$$
x^{\prime}=\left(x_{\eta 0}: \eta \in\{0,1\}^{d-1}\right) \text { and } \quad x^{\prime \prime}=\left(x_{\eta 1}: \eta \in\{0,1\}^{d-1}\right) .
$$

By construction, the images of $\mu^{*}$ under the projections $x \mapsto x^{\prime}$ and $x \mapsto x^{\prime \prime}$ are equal to the measure $\mu_{d-1}$ associated to the transformations $T_{1}, \ldots, T_{d-1}$. We also remark that

$$
T_{d}^{\Delta} T_{d}^{*-1}=\mathrm{Id} \times T_{d}^{\circ}, \quad \text { where } \quad T_{d}^{\circ}=\underbrace{T_{d} \times \cdots \times T_{d}}_{2^{d-1} \text { times }} .
$$

From the inductive definition of the measure $\mu^{*}$, we deduce: 
Lemma 3. Let $F \in L^{\infty}\left(\mu^{*}\right)$ be a function invariant under the transformation $T_{d}^{\Delta} T_{d}^{*-1}$. Then there exists a function $G$ on $X^{2^{d-1}}$, belonging to $L^{\infty}\left(\mu_{d-1}\right)$, such that

$$
F(x)=G\left(x^{\prime}\right) \quad \text { for } \mu^{*} \text {-almost every } x=\left(x^{\prime}, x^{\prime \prime}\right) \in X^{*} .
$$

By induction on $d$, we have:

COROLlary 1. Let $F \in L^{\infty}\left(\mu^{*}\right)$ be a function that is invariant under the transformations $T_{i}^{\Delta} T_{d}^{*-1}$ for $i=1, \ldots, d$. Then there exists a function $f \in L^{\infty}(\mu)$ such that $F(x)=f\left(x_{\emptyset}\right)$ for $\mu^{*}$-almost every $x \in X^{*}$.

We write $X^{\sharp}=X^{2^{d}-1}$ and identify $X^{*}$ with $X \times X^{\sharp}$ by isolating the coordinate $\emptyset$ of each point: each point $x \in X^{*}$ is written

$$
x=\left(x_{\emptyset}, x^{\sharp}\right), \quad \text { where } \quad x^{\sharp}=\left(x_{\epsilon}: \epsilon \in\{0,1\}^{d}, \epsilon \neq \emptyset\right) \in X^{\sharp} .
$$

We write $\mu^{\sharp}$ for the image of $\mu^{*}$ in $X^{\sharp}$ under the projection $x \mapsto x^{\sharp}$.

For $1 \leq i \leq d$, the measure preserving transformation $T_{i}^{\Delta} T_{i}^{*-1}$ of $\left(X^{*}, \mu^{*}\right)$ leaves the coordinate $x_{\emptyset}$ of each point $x$ invariant, and thus we can write this transformation as

$$
T_{i}^{\Delta} T_{i}^{*-1}=\operatorname{Id}_{X} \times T_{i}^{\sharp},
$$

where $T_{i}^{\sharp}$ is the measure preserving transformation of $\left(X^{\sharp}, \mu^{\sharp}\right)$ given by

$$
\left(T_{i} x^{\sharp}\right)_{\epsilon}=\left\{\begin{array}{ll}
T_{i} x_{\epsilon} & \text { if } \epsilon_{i}=1, \\
x_{\epsilon} & \text { if } \epsilon_{i}=0,
\end{array} \quad \text { for } \emptyset \neq \epsilon \in\{0,1\}^{d} .\right.
$$

By Corollary 1 we immediately deduce:

Corollary 2. Let $\mathcal{J}^{\sharp}$ be the $\sigma$-algebra of invariant sets of $\left(X^{\sharp}, \mu^{\sharp}\right.$, $\left.T_{1}^{\sharp}, \ldots, T_{d}^{\sharp}\right)$. Then for every $A \in \mathcal{J}^{\sharp}$ there exists a subset $B$ of $X$ with

$$
\mathbf{1}_{B}\left(x_{\emptyset}\right)=\mathbf{1}_{A}\left(x^{\sharp}\right) \quad \text { for } \mu^{*} \text {-almost every } x=\left(x_{\emptyset}, x^{\sharp}\right) \in X^{*} .
$$

We remark that conversely, if $A \subset X^{\sharp}$ and $B \subset X$ satisfy (14), then $A$ is invariant under $T_{i}^{\sharp}$ for every $i$.

Lemma 4 ([HK1, Lemma 4.3]). Let $\mathcal{Z}$ be the $\sigma$-algebra on $X$ consisting of sets $B$ such that there exists a subset $A$ of $X^{\sharp}$ satisfying the relation (14) of Corollary 2. Then for every $f \in L^{\infty}(\mu)$ we have

$$
\|f\|=0 \quad \text { if and only if } \quad \mathbb{E}_{\mu}(f \mid \mathcal{Z})=0 .
$$

Proof. Assume first that $\mathbb{E}_{\mu}(f \mid \mathcal{Z})=0$. Let $F$ be the function on $X^{\sharp}$ given by

$$
F\left(x^{\sharp}\right)=\prod_{\emptyset \neq \epsilon \in\{0,1\}^{d}} f\left(x_{\epsilon}\right) .
$$

Let $\mathcal{J}^{\sharp}$ be defined as in Corollary 2. The function $x \mapsto \mathbb{E}_{\mu^{\sharp}}\left(F \mid \mathcal{J}^{\sharp}\right)\left(x^{\sharp}\right)$ on $X^{*}$ is invariant under all transformations $T_{i}^{\Delta} T_{i}^{*-1}$. Thus by Corollary 1 , 
there exists a function $g$ on $X$ with

$$
g\left(x_{\emptyset}\right)=\mathbb{E}_{\mu^{\sharp}}\left(F \mid \mathcal{J}^{\sharp}\right)\left(x^{\sharp}\right) \quad \text { for } \mu^{*} \text {-almost every } x=\left(x_{\emptyset}, x^{\sharp}\right) .
$$

We remark that the function $g$ is measurable with respect to $\mathcal{Z}$ by definition of this $\sigma$-algebra. Since $\mu^{*}$ is invariant under $\operatorname{Id}_{X} \times T_{i}^{\sharp}$ for every $i$, by definition of the seminorm we have

$$
\begin{aligned}
\|f\|^{2^{d}} & =\int_{X^{*}} f\left(x_{\emptyset}\right) F\left(x^{\sharp}\right) d \mu^{*}\left(x_{\emptyset}, x^{\sharp}\right) \\
& =\int_{X^{*}} f\left(x_{\emptyset}\right) \mathbb{E}_{\mu^{\sharp}}\left(F \mid \mathcal{J}^{\sharp}\right)\left(x^{\sharp}\right) d \mu^{*}(x)=\int_{X} f\left(x_{\emptyset}\right) g\left(x_{\emptyset}\right) d \mu\left(x_{\emptyset}\right)=0 .
\end{aligned}
$$

We assume now that $\|f\|=0$. Let $g \in L^{\infty}(\mu)$ be measurable with respect to $\mathcal{Z}$. By definition, there exists a function $G \in L^{\infty}\left(\mu^{\sharp}\right)$ with $g\left(x_{\emptyset}\right)=G\left(x^{\sharp}\right)$, $\mu^{*}$-almost everywhere. We have

$$
\int_{X} f(x) g(x) d \mu(x)=\int_{X^{*}} f\left(x_{\emptyset}\right) G\left(x^{\sharp}\right) d \mu^{*}\left(x_{\emptyset}, x^{\sharp}\right)
$$

and using the bound (12) of Proposition 2 we deduce that this integral is equal to zero.

In the case of a single transformation, the $\sigma$-algebra $\mathcal{Z}$ is the $\sigma$-algebra $\mathcal{Z}_{d-1}$ of [HK1], where it is shown that the corresponding factor $Z_{d-1}$ has the structure of an inverse limit of $(d-1)$-step nilsystems. In the present case of several transformations, $\mathcal{Z}$ apparently only has a weaker structure and we stop following [HK1] at this point.

\section{Proof of Theorem 2}

3.1. The system $\left(X^{*}, \mu^{*}, T_{1}^{*}, \ldots, T_{d}^{*}\right)$. Let $\mathcal{X}^{\sharp}$ be the $\sigma$-algebra on $X^{*}$ corresponding to the factor $X^{\sharp}$ of $X^{*} ; \mathcal{X}^{\sharp}$ is spanned by the projections $x \mapsto x_{\epsilon}: X^{*} \rightarrow X$ for $\epsilon \in\{0,1\}^{d}, \epsilon \neq \emptyset$.

Lemma 5. The subspace of $L^{2}\left(\mu^{*}\right)$ consisting of functions with zero conditional expectation on $\mathcal{X}^{\sharp}$ is the space spanned by the functions of the form

$$
F(x)=\prod_{\epsilon \in\{0,1\}^{d}} f_{\epsilon}\left(x_{\epsilon}\right), \quad \text { where }\left|f_{\epsilon}\right| \leq 1 \text { for all } \epsilon \text { and } \mathbb{E}_{\mu}\left(f_{\emptyset} \mid \mathcal{Z}\right)=0 .
$$

Proof. Let $\mathcal{L}$ be the closed subspace of $L^{2}\left(\mu^{*}\right)$ spanned by the functions of the type given in the statement and let $\mathcal{L}^{\prime}$ be the closed subspace of $L^{2}\left(\mu^{*}\right)$ spanned by the functions of the form

$$
F^{\prime}(x)=\prod_{\epsilon \in\{0,1\}^{d}} f_{\epsilon}^{\prime}\left(x_{\epsilon}\right), \quad \text { where }\left|f_{\epsilon}^{\prime}\right| \leq 1 \text { for all } \epsilon \text { and } f_{\emptyset}^{\prime} \text { is } \mathcal{Z} \text {-measurable. }
$$

The sum of these spaces is clearly dense in $L^{2}\left(X^{*}, \mu^{*}\right)$. We claim that they are orthogonal. 
Let $f_{\epsilon}$ and $f_{\epsilon}^{\prime}, \epsilon \in\{0,1\}^{d}$, be as above. For every $i$, the function $x \mapsto$ $f_{\emptyset}\left(x_{\emptyset}\right) f_{\emptyset}^{\prime}\left(x_{\emptyset}\right)$ is invariant under $\operatorname{Id}_{X} \times T_{i}^{\sharp}$ and thus

$$
\int f_{\emptyset}\left(x_{\emptyset}\right) f_{\emptyset}^{\prime}\left(x_{\emptyset}\right) \prod_{\emptyset \neq \epsilon \in\{0,1\}^{d}} f\left(x_{\epsilon}\right) f^{\prime}\left(x_{\epsilon}\right) d \mu^{*}=\int f_{\emptyset}\left(x_{\emptyset}\right) f_{\emptyset}^{\prime}\left(x_{\emptyset}\right) G\left(x^{\sharp}\right) d \mu^{*},
$$

where

$$
G=\mathbb{E}_{\mu^{\sharp}}\left(\bigotimes_{\emptyset \neq \epsilon \in\{0,1\}^{d}} f_{\epsilon} f_{\epsilon}^{\prime} \mid \mathcal{J}^{\sharp}\right) .
$$

By Corollary 2, there exists a function $g \in L^{\infty}(\mu)$, measurable with respect to $\mathcal{Z}$, with $g\left(x_{\emptyset}\right)=G\left(x^{\sharp}\right)$ for $\mu^{*}$-almost every $x=\left(x_{\emptyset}, x^{\sharp}\right)$ and so the integral above is equal to

$$
\int f_{\emptyset}\left(x_{\emptyset}\right) f_{\emptyset}^{\prime}\left(x_{\emptyset}\right) g\left(x_{\emptyset}\right) d \mu\left(x_{\emptyset}\right) .
$$

Since $\mathbb{E}_{\mu}\left(f_{\emptyset} \mid \mathcal{Z}\right)=0$ and the function $f_{\emptyset}^{\prime} \cdot g$ is $\mathcal{Z}$-measurable, the above integral vanishes and thus $\mathcal{L}$ and $\mathcal{L}^{\prime}$ are orthogonal. Therefore $\mathcal{L}$ is the orthogonal complement to $\mathcal{L}^{\prime}$.

On the other hand, $\mathcal{L}^{\prime}$ clearly contains $L^{2}\left(X^{*}, \mathcal{X}^{\sharp}, \mu^{*}\right)$ and by the definition of $\mathcal{Z}$ in Lemma 4 we have the opposite inclusion. Thus these spaces are equal. Therefore, $\mathcal{L}$ is the orthogonal complement to $L^{2}\left(X^{*}, \mathcal{X}^{\sharp}, \mu^{*}\right)$, and this is the announced result.

3.2. Iterating the construction. We now define a new system $\left(X^{* *}, \mu^{* *}\right.$, $\left.T_{1}^{* *}, \ldots, T_{d}^{* *}\right)$ where $X^{* *}:=\left(X^{*}\right)^{*}=\left(X^{2^{d}}\right)^{2^{d}}$. It is built from the system $\left(X^{*}, \mu^{*}, T_{1}^{*}, \ldots, T_{d}^{*}\right)$ in the same way that $\left(X^{*}, \mu^{*}, T_{1}^{*}, \ldots, T_{d}^{*}\right)$ was built from $\left(X, \mu, T_{1}, \ldots, T_{d}\right)$. The points of $X^{* *}$ are written

$$
x=\left(x_{\epsilon \eta}: \epsilon, \eta \in\{0,1\}^{d}\right),
$$

with the $2^{d}$ natural projections $\pi_{\eta}^{*}: X^{* *} \rightarrow X^{*}$ being given by

$$
\left(\pi_{\eta}^{*}(x)\right)_{\epsilon}=x_{\epsilon \eta} .
$$

The seminorm $\|\cdot\|^{*}$ on $L^{\infty}\left(\mu^{*}\right)$ is defined from the measure $\mu^{* *}$ in the same way that the seminorm $\|\cdot\|$ on $L^{\infty}(\mu)$ was defined from the measure $\mu^{*}$.

LEMMA 6. Let

$$
F(x)=\prod_{\epsilon \in\{0,1\}^{d}} f_{\epsilon}\left(x_{\epsilon}\right), \quad \text { where } f_{\epsilon} \in L^{\infty}(\mu) \text { for all } \epsilon \text { and }\left\|f_{\emptyset}\right\|=0 .
$$

Then $\|F\|^{*}=0$.

Proof. We can assume that $\left|f_{\epsilon}\right| \leq 1$ for $\epsilon \neq \emptyset$. By Lemma 1 applied to the measure $\mu^{* *},\left(\|F\|^{*}\right)^{2^{d}}$ is equal to

$$
\lim _{P_{d} \rightarrow \infty} \ldots \lim _{P_{1} \rightarrow \infty} \frac{1}{P_{d} \ldots P_{1}} \sum_{\substack{0 \leq p_{d}<P_{d} \\ 0 \leq p_{1}<P_{1}}} I\left(p_{1}, \ldots, p_{d}\right),
$$


where

$$
I\left(p_{1}, \ldots, p_{d}\right):=\int \prod_{\eta \in\{0,1\}^{d}} T_{1}^{*\left(1-\eta_{1}\right) p_{1}} \ldots T_{d}^{*\left(1-\eta_{d}\right) p_{d}}\left(\bigotimes_{\epsilon \in\{0,1\}^{d}} f_{\epsilon}\right) d \mu^{*} .
$$

By definition of $T_{i}^{*}$, this integral is equal to

$$
\int \bigotimes_{\epsilon \in\{0,1\}^{d}}\left(\prod_{\eta \in\{0,1\}^{d}} T_{1}^{\left(1-\eta_{1}\right)\left(1-\epsilon_{1}\right) p_{1}} \ldots T_{d}^{\left(1-\eta_{d}\right)\left(1-\epsilon_{d}\right) p_{d}} f_{\epsilon}\right) d \mu^{*}
$$

By Lemma 1 again, but now applied to the measure $\mu^{*},\left(\|F\|^{*}\right)^{2^{d}}$ is equal to

$$
\begin{aligned}
\lim _{P_{d} \rightarrow \infty} \ldots \lim _{P_{1} \rightarrow \infty} \frac{1}{P_{d} \ldots P_{1}} & \lim _{N_{d} \rightarrow \infty} \ldots \lim _{N_{1} \rightarrow \infty} \frac{1}{N_{d} \ldots N_{1}} \\
& \times \sum_{\substack{0 \leq p_{d}<P_{d} \\
0 \leq p_{1}<P_{1}}} \sum_{\substack{0 \leq n_{d}<N_{d} \\
0 \leq n_{1}<N_{1}}} J\left(n_{1}, \ldots, n_{d}, p_{1}, \ldots, p_{d}\right),
\end{aligned}
$$

where

$$
\begin{aligned}
J\left(n_{1}, \ldots, n_{d}, p_{1}, \ldots, p_{d}\right) & \\
& :=\int \prod_{\epsilon, \eta \in\{0,1\}^{d}} T_{1}^{\left(1-\epsilon_{1}\right)\left(1-\eta_{1}\right) p_{1}+\left(1-\epsilon_{1}\right) n_{1}} \ldots T_{d}^{\left.\left(1-\epsilon_{d}\right)\left(1-\eta_{d}\right) p_{d}+\left(1-\epsilon_{d}\right) n_{d}\right)} f_{\epsilon} d \mu .
\end{aligned}
$$

At this point, it is more convenient to identify $\{0,1\}^{d}$ with the family of subsets of $[d]$. Let $\theta \subset[d]$. In the product in $\epsilon$ and $\eta$ of the last formula, we gather all terms with $\epsilon \cup \eta=\theta$. For $1 \leq i \leq d$ we have $\left(1-\epsilon_{i}\right)\left(1-\eta_{i}\right) p_{i}+$ $\left(1-\epsilon_{i}\right) n_{i}=\left(1-\theta_{i}\right)\left(p_{i}+n_{i}\right)+\eta_{i} n_{i}$. Thus

$$
\begin{aligned}
J\left(n_{1}, \ldots, n_{d}, p_{1}, \ldots, p_{d}\right) & =\int \prod_{\theta \subset[d]} T_{1}^{\left(1-\theta_{1}\right)\left(p_{1}+n_{1}\right)} \ldots T_{d}^{\left(1-\theta_{d}\right)\left(p_{d}+n_{d}\right)} g_{\theta}^{\left(n_{1}, \ldots, n_{d}\right)} d \mu,
\end{aligned}
$$

where

$$
g_{\theta}^{\left(n_{1}, \ldots, n_{d}\right)}=\prod_{\eta \subset \theta} T_{1}^{\eta_{1} n_{1}} \ldots T_{d}^{\eta_{d} n_{d}} \prod_{\epsilon \in \epsilon \cup \eta=\theta} f_{\epsilon} .
$$

We consider $P_{1}, \ldots, P_{d}$ as fixed. We have

$$
\begin{aligned}
K\left(n_{1}, \ldots, n_{d}\right):=\frac{1}{P_{d} \ldots P_{1}} \sum_{\substack{0 \leq p_{d}<P_{d} \\
0 \leq p_{1}<P_{1}}} J\left(n_{1}, \ldots, n_{d}, p_{1}, \ldots, p_{d}\right) \\
=\frac{1}{P_{d} \ldots P_{1}} \sum_{\substack{0 \\
n_{d} \leq p_{d}<n_{d}+P_{d} \\
n_{1} \leq p_{1}<n_{1}+P_{1}}} \int \prod_{\theta \subset[d]} T_{1}^{\left(1-\theta_{1}\right) p_{1} \ldots T_{d}^{\left(1-\theta_{d}\right) p_{d}} g_{\theta}^{\left(n_{1}, \ldots, n_{d}\right)} d \mu .}
\end{aligned}
$$


We remark that for all $n_{1}, \ldots, n_{d}$ we have

$$
\left|g_{\theta}^{\left(n_{1}, \ldots, n_{d}\right)}\right| \leq 1 \quad \text { for every } \theta \text { and } \quad g_{\emptyset}^{\left(n_{1}, \ldots, n_{d}\right)}=f_{\emptyset} .
$$

Therefore, by Lemma 2, for every $\delta>0$ there exists $P$ such that

$$
\left|K\left(n_{1}, \ldots, n_{d}\right)\right|<\delta \text { for all } n_{1}, \ldots, n_{d} \text { whenever } P_{1}, \ldots, P_{d}>P,
$$

and the announced conclusion follows.

3.3. End of the proof. We recall that $\mathcal{W}^{*}$ is the $\sigma$-algebra

$$
\mathcal{W}^{*}=\bigvee_{i=1}^{d} \mathcal{I}\left(T_{i}^{*}\right)
$$

on $\left(X^{*}, \mu^{*}\right)$. We show that if a function $F \in L^{\infty}\left(\mu^{*}\right)$ satisfies $\mathbb{E}_{\mu^{*}}\left(F \mid \mathcal{W}^{*}\right)$ $=0$ then $\|F\|^{*}=0$.

For every $\epsilon \neq \emptyset$, there exists $i \in\{1, \ldots, d\}$ with $\epsilon_{i}=1$ and the projection $x \mapsto x_{\epsilon}$ is invariant under $T_{i}^{*}$ and thus is $\mathcal{W}^{*}$-measurable. Hence $\mathcal{X}^{\sharp} \subset \mathcal{W}^{*}$. Thus $\mathbb{E}_{\mu^{*}}\left(F \mid \mathcal{X}^{\sharp}\right)=0$.

Therefore, by Lemma 5 we can restrict to the case that

$$
F(x)=\prod_{\epsilon \in\{0,1\}^{d}} f_{\epsilon}\left(x_{\epsilon}\right) \quad \text { where }\left|f_{\epsilon}\right| \leq 1 \text { for all } \epsilon \text { and } \mathbb{E}_{\mu}\left(f_{\emptyset} \mid \mathcal{Z}\right)=0 .
$$

We have $\left\|f_{\emptyset}\right\|=0$ by Lemma 4 , and Lemma 6 yields $\|F\|^{*}=0$.

4. Changing the order of the transformations. The next proposition shows that we can exchange the order of the limits in the formula (9) of Lemma 1. This result is parallel to Proposition 3.7 of [HK1], but we cannot simply copy its proof, as it depends on formula (9) of [HK1] which has no analog in the present context. It seems that here we need some technology, for example the "modules" of [CL] and/or [FW]. This is the only point in this paper where we need more elaborate tools.

Proposition 3. Let $\sigma$ be a permutation of $[d], \sigma_{*}$ the permutation of $\{0,1\}^{d}$ given by $\left(\sigma_{*}(\epsilon)\right)_{i}=\epsilon_{\sigma(i)}$ for every $i$, and $\Sigma$ the associated permutation of $X^{*}$, given by

$$
(\Sigma x)_{\epsilon}=x_{\sigma_{*}(\epsilon)} \quad \text { for every } \epsilon \in\{0,1\}^{d} .
$$

Then the box measure associated to the transformations $T_{\sigma(1)}, \ldots, T_{\sigma(d)}$ is the image under $\Sigma$ of the box measure associated to the transformations $T_{1}, \ldots, T_{d}$.

We immediately deduce:

COROllary 3. The seminorm $\|\cdot\|_{T_{1}, \ldots, T_{d}}$ remains unchanged if the transformations $T_{1}, \ldots, T_{d}$ are permuted. 
4.1. Proof of Proposition 3, first step. First we check that it suffices to prove the result for the case of two transformations.

Indeed, any permutation of $\{1, \ldots, d\}$ can be written as a product of transpositions of two consecutive terms and we can thus assume that $\sigma$ is the transposition of $i$ and $i+1$ for some $i$ with $1 \leq i<d$.

Fix $i$ and let $\tau$ be the box measure associated to $T_{1}, \ldots, T_{i-1}$ (or equal to $\mu$ if $i=1$ ), $S_{1}=T_{i} \times \cdots \times T_{i}$, and $S_{2}=T_{i+1} \times \cdots \times T_{i+1}$. Applying the result for these transformations we find that the box measure associated to $T_{1}, \ldots, T_{i-1}, T_{i+1}, T_{i}$ is equal to the image of the box measure associated to $T_{1}, \ldots, T_{i-1}, T_{i}, T_{i+1}$ under the permutation of the last two digits. We immediately deduce the announced result.

Henceforth we assume that $d=2$. We write $\mu_{2}$ for the box measure associated to $T_{1}$ and $T_{2}$ and $\mu_{2}^{\prime}$ for the measure associated to $T_{2}$ and $T_{1}$. We want to show that $\mu_{2}^{\prime}$ is the image of $\mu_{2}$ under the map

$$
\left(x_{00}, x_{01}, x_{10}, x_{11}\right) \mapsto\left(x_{00}, x_{10}, x_{01}, x_{11}\right): X^{4} \rightarrow X^{4} .
$$

We recall that

$$
\begin{aligned}
& \mu^{*}=\left(\mu \times_{\mathcal{I}\left(T_{1}\right)} \mu\right) \times_{\mathcal{I}\left(T_{2} \times T_{2}\right)}\left(\mu \times_{\mathcal{I}\left(T_{1}\right)} \mu\right), \\
& \mu^{\circ}=\left(\mu \times_{\mathcal{I}\left(T_{2}\right)} \mu\right) \times_{\mathcal{I}\left(T_{1} \times T_{1}\right)}\left(\mu \times_{\mathcal{I}\left(T_{2}\right)} \mu\right) .
\end{aligned}
$$

4.2. Reduction to the ergodic case. We check that we can restrict to the case that $\left(X, \mu, T_{1}, T_{2}\right)$ is ergodic. Indeed, let $\mathcal{J}$ be the $\sigma$-algebra of sets invariant under $T_{1}$ and $T_{2}$ and let

$$
\mu=\int \mu_{\omega} d P(\omega)
$$

be the ergodic decomposition of $\mu$ under the action of $T_{1}$ and $T_{2}$. Since $\mathcal{J} \subset \mathcal{I}\left(T_{1}\right)$ we have

$$
\mu \times_{\mathcal{I}\left(T_{1}\right)} \mu=\int\left(\mu_{\omega} \times_{\mathcal{I}\left(T_{1}\right)} \mu_{\omega}\right) d P(\omega) .
$$

Since $\mathcal{J} \otimes \mathcal{J} \subset \mathcal{I}\left(T_{2} \times T_{2}\right)$, by definition of $\mu^{*}$, we have

$$
\mu^{*}=\int\left(\left(\mu_{\omega} \times_{\mathcal{I}\left(T_{1}\right)} \mu_{\omega}\right) \times_{\mathcal{I}\left(T_{2} \times T_{2}\right)}\left(\mu_{\omega} \times_{\mathcal{I}\left(T_{1}\right)} \mu_{\omega}\right)\right) d P(\omega)
$$

and a similar expression holds for $\mu^{\circ}$. Applying the result to the ergodic measures $\mu_{\omega}$ we deduce the general case.

Henceforth we assume that $\left(X, \mu, T_{1}, T_{2}\right)$ is ergodic.

4.3. Decomposition. Let $f_{00}, f_{01}, f_{10}, f_{11} \in L^{\infty}(\mu)$. We want to show that

$$
\begin{aligned}
& \int f_{00}\left(x_{00}\right) f_{01}\left(x_{01}\right) f_{10}\left(x_{10}\right) f_{11}\left(x_{11}\right) d \mu^{*}\left(x_{00}, x_{01}, x_{10}, x_{11}\right) \\
& \quad=\int f_{00}\left(x_{00}\right) f_{01}\left(x_{10}\right) f_{10}\left(x_{01}\right) f_{11}\left(x_{11}\right) d \mu^{\circ}\left(x_{00}, x_{01}, x_{10}, x_{11}\right) .
\end{aligned}
$$


Let $\mathcal{Y}$ be the $\sigma$-algebra on $X$ corresponding to the maximal isometric extension of $\left(X, \mathcal{I}\left(T_{1}\right), \mu, T_{2}\right)$ in $\left(X, \mu, T_{2}\right)$ and let $\mathcal{Y}^{\prime}$ be the $\sigma$-algebra on $X$ corresponding to the maximal isometric extension of $\left(X, \mathcal{I}\left(T_{2}\right), \mu, T_{1}\right)$ in $\left(X, \mu, T_{1}\right)\left({ }^{3}\right)$.

For every $\epsilon>0$, we can write $f_{00}$ as a sum $f_{00}=f+f^{\prime}+g+h$ of four bounded functions where $f$ is measurable with respect to $\mathcal{Y}, f^{\prime}$ is measurable with respect to $\mathcal{Y}^{\prime}, \mathbb{E}_{\mu}(g \mid \mathcal{Y})=\mathbb{E}_{\mu}\left(g \mid \mathcal{Y}^{\prime}\right)=0$ and $\|h\|_{2}<\epsilon$. Therefore, we are reduced to considering three different cases: the case that $f_{00}$ is measurable with respect to $\mathcal{Y}$, the completely similar case that $f_{00}$ is measurable with respect to $\mathcal{Y}^{\prime}$, and the case that $\mathbb{E}_{\mu}\left(f_{00} \mid \mathcal{Y}\right)=\mathbb{E}_{\mu}\left(f_{00} \mid \mathcal{Y}^{\prime}\right)=0$.

4.4. The case that $f_{00}$ is measurable with respect to $\mathcal{Y}$

Lemma 7. Assume that $f_{00}$ is measurable with respect to $\mathcal{Y}$. Then

$$
\sup _{m \in \mathbb{Z}}\left\|\frac{1}{N} \sum_{n=0}^{N-1} T_{1}^{n}\left(T_{2}^{m} f_{00} \cdot f_{10}\right)-\mathbb{E}_{\mu}\left(T_{2}^{m} f_{00} \cdot f_{10} \mid \mathcal{I}\left(T_{1}\right)\right)\right\|_{L^{2}(\mu)} \rightarrow 0
$$

as $N \rightarrow \infty$.

Proof. We use the vocabulary of "modules" as in [CL]. We can restrict to the case that $f_{00}=\phi_{i}$ where $\left(\phi_{1}, \ldots, \phi_{k}\right)$ is a base of a $\left(\mathcal{I}\left(T_{1}\right), T_{2}\right)$-module and $1 \leq i \leq k$ : there exists an $\mathcal{I}\left(T_{1}\right)$-measurable map $x \mapsto U(x)$ with values in the group of unitary $k \times k$ matrices such that

$$
T_{2} \phi_{i}=\sum_{j=1}^{k} U_{i, j} \cdot \phi_{j}
$$

For every $m$,

$$
\mathbb{E}_{\mu}\left(T_{2}^{m} f_{00} \cdot f_{10} \mid \mathcal{I}\left(T_{1}\right)\right)=\sum_{j=1}^{k} U_{i, j}^{(m)} \cdot \mathbb{E}_{\mu}\left(\phi_{j} f_{10} \mid \mathcal{I}\left(T_{1}\right)\right),
$$

where $U^{(m)}$ denotes the iterated cocycle:

$$
U^{(m)}(x)=U\left(T_{2}^{m-1} x\right) \ldots U\left(T_{2} x\right) U(x) .
$$

For every $n$,

$$
T_{1}^{n}\left(T_{2}^{m} f_{00} \cdot f_{10}\right)=\sum_{j=1}^{k} U_{i, j}^{(m)} \cdot T_{1}^{n}\left(\phi_{j} f_{10}\right)
$$

$\left({ }^{3}\right)$ In fact, these two $\sigma$-algebras are equal, but we do not prove equality here. 
Thus for every $N$ we have

$$
\begin{gathered}
\left\|\frac{1}{N} \sum_{n=0}^{N-1} T_{1}^{n}\left(T_{2}^{m} f_{00} \cdot f_{10}\right)-\mathbb{E}_{\mu}\left(T_{2}^{m} f_{00} \cdot f_{10} \mid \mathcal{I}\left(T_{1}\right)\right)\right\|_{L^{2}(\mu)} \\
\leq \sum_{j=1}^{k}\left\|\frac{1}{N} \sum_{n=0}^{N-1} T_{2}^{n}\left(\phi_{j} f_{10}\right)-\mathbb{E}_{\mu}\left(\phi_{j} \cdot f_{10} \mid \mathcal{I}\left(T_{1}\right)\right)\right\|_{L^{2}(\mu)} .
\end{gathered}
$$

We now prove formula (17) when $f_{00}$ is measurable with respect to $\mathcal{Y}$. By Lemma 1, the left hand side of (17) is equal to

$$
\lim _{M \rightarrow \infty} \frac{1}{M} \sum_{m=0}^{M-1} \int_{N \rightarrow \infty} \lim _{N} \frac{1}{N} \sum_{n=0}^{N-1} T_{1}^{n}\left(T_{2}^{m} f_{00} \cdot f_{10}\right) \cdot\left(T_{2}^{m} f_{01} \otimes f_{11}\right) d \mu .
$$

By Lemma 7, the limit as $N \rightarrow \infty$ in this expression is uniform in $M$, thus the two limits can be permuted and the above expression can be rewritten as

$$
\lim _{N \rightarrow \infty} \frac{1}{N} \sum_{n=0}^{N-1} \int_{M \rightarrow \infty} \lim _{M} \frac{1}{M} \sum_{m=0}^{M-1} T_{2}^{m}\left(T_{1}^{n} f_{00} \cdot f_{01}\right) \cdot\left(T_{1}^{n} f_{10} \cdot f_{11}\right) d \mu,
$$

which is equal to the right hand side of (17).

4.5. The case that $\mathbb{E}_{\mu}\left(f_{00} \mid \mathcal{Y}\right)=\mathbb{E}_{\mu}\left(f_{00} \mid \mathcal{Y}^{\prime}\right)=0$. It is shown in [CL] that the $T_{2} \times T_{2}$ invariant $\sigma$-algebra of $\left(X \times X, \mu \times \times_{\mathcal{I}\left(T_{1}\right)} \mu\right)$ is included in $\mathcal{Y} \otimes \mathcal{Y}$. Since $\mathbb{E}_{\mu}\left(f_{00} \mid \mathcal{Y}\right)=0$, we have $\mathbb{E}_{\mu \times{ }_{\mathcal{I}\left(T_{1}\right)}}\left(f_{00} \otimes f_{01} \mid \mathcal{I}\left(T_{2} \times T_{2}\right)\right)=0$ and, by the definition (15) of $\mu^{*}$, the left hand side of (17) is equal to zero. By the same reasoning, the right hand side is also equal to zero.

\section{References}

[A] T. Austin, On the norm convergence of nonconventional ergodic averages, Ergodic Theory Dynam. Systems, to appear.

[BHK] V. Bergelson, B. Host and B. Kra (with an appendix by I. Ruzsa), Multiple recurrence and nilsequences, Invent. Math. 160 (2005), 261-303.

[CL] J.-P. Conze and E. Lesigne, Théorèmes ergodiques pour des mesures diagonales, Bull. Soc. Math. France 112 (1984), 143-175.

[FHK] N. Frantzikinakis, B. Host and B. Kra, Multiple recurrence and convergence for sequences related to the prime numbers, J. Reine Angew. Math. 611 (2007), 131144.

[FK1] N. Frantzikinakis and B. Kra, Convergence of multiple ergodic averages for some commuting transformations, Ergodic Theory Dynam. Systems 25 (2005), 799809 .

[FK2] - - , Polynomial averages converge to the product of the integrals, Israel J. Math. 148 (2005), 267-276.

[F] H. Furstenberg, Ergodic behavior of diagonal measures and a theorem of Szemerédi on arithmetic progressions, J. Anal. Math. 31 (1977), 204-256. 
[FW] H. Furstenberg and B. Weiss, A mean ergodic theorem for $\frac{1}{N} \sum_{n=1}^{n} f\left(T^{n} x\right) g\left(T^{n^{2}} x\right)$, in: Convergence in Ergodic Theory and Probability, V. Bergelson et al. (eds.), Ohio State Univ. Math. Res. Inst. Publ. 5, de Gruyter, Berlin, 1996, 193-227.

[HK1] B. Host and B. Kra, Nonconventional ergodic averages and nilmanifolds, Ann. of Math. 161 (2005), 397-488.

[HK2] - - - Convergence of polynomial ergodic averages, Israel J. Math. 149 (2005), $1-19$.

[L] A. Leibman, Convergence of multiple ergodic averages along polynomials of several variables, ibid. 146 (2005), 303-316.

[T] T. Tao, Norm convergence of multiple ergodic averages for commuting transformations, Ergodic Theory Dynam. Systems 28 (2008), 657-688.

[To] H. Towsner, Convergence of diagonal ergodic averages, ibid., to appear.

Laboratoire d'Analyse et de Mathématiques Appliquées

UMR CNRS 8050

Université Paris-Est

5 bd Descartes

77454 Marne-la-Vallée Cedex 2, France

E-mail: bernard.host@univ-mlv.fr

Received October 17, 2008

Revised version April 10, 2009 